Anthropology and Aging Comprehensive
Reviews

Edited by Robert L Rubinstein, 203 pages, Dordrecht, The Netherlands, Dfl130.00, US\$74.00, $£ 47.00$, Kluwer Academic Publishers, 1990

This book is the result of a project of the Association of Anthropology and Aging which is dedicated to 'exploration and understanding of aging within and across the diversity of human cultures'. This is a tall order for a slim volume, but the title is accurate in presenting it as 'comprehensive reviews' because that is what each of the seven chapters represents.

Each chapter is by an anthropologist, a gerontologist or, in one case, by a nurse. There are extensive bibliographies much of which refer to recent work; some, indeed, is described as 'forthcoming' which I interpret as 'not quite yet accepted for publication'.

Despite the immensity of the task, the authors have managed to cover a very large range of topics from descriptions of anthropological methodology applied to gerontology and factors affecting life-span, to the effects of decollectivisation (sic) in recent Chinese history.

Fascinating views are given of recent work on longevity and maximum lifespan, including methods of determining the age at death of individuals whose fossil remains have been uncovered. It is concluded that from Neanderthal man onwards 'no special factors need to be invoked to account for longevity of modern humans'. This suggests that if these hominids enjoyed the environment now lived in by modern Europeans, they would have the same longevity. The biological significance of the menopause is briefly discussed and it is pointed out that menstruation was for most women probably a very rare event since most of their adult lives were spent either pregnant or in a state of lactation amenorrhoea.

Several of the authors emphasise that ageing can only be properly studied in the context of the individual's total lifeexperience, which is certainly true.

The nurse points out that since nurses spend such prolonged periods with their elderly charges they possess the same chance to study and observe as do anthropologists, and this underlines the potential contribution we may expect from this profession throughout the world.

I greatly enjoyed the chapters on old age in Japan and China. It is emphasised that we must realise the true significance of 'form' and 'meaning', for example the use of honorific titles and bowing to elders does not necessarily indicate that their status or security are greater than in areas where such observances are absent.

In the context of China it is suggested that the widespread occurrence of three-generation households may have much more to do with housing shortages and economic necessity than with special reverence for the aged, who in many instances are essential for the household economy as child-minders and performers of chores where son and daughter both have to work outside the house.

It is heartening to read about so much research now being planned in the field of ageing as a part of life-development in many ethnic and cultural settings.

This volume offers a great deal of information and indicates almost endless opportunities for further research worldwide.

J WILLIAMSON, Professor Emeritus,

University of Edinburgh, Scotland.

\section{Beyond Baby M: Ethical Issues in New Reproductive Techniques}

D M Bartels, R Priester, D E Vawter and A L Caplan, 288 pages, New Jersey, £30.10 hc, Humana Press, 1990

I do not like to think of myself as a 'waspish' person but I find myself reacting waspishly to this book. This is partly because I looked forward so much to reading it when asked to provide a review, only to have my anticipated pleasure severely dashed, and partly because it is not what its title proclaims it to be. The book has a reasonable pedigree coming as it does as a volume in the series, Contemporary Issues in Biomedicine, Ethics and Society, but it fails to impress. It is a collection of short, superficial and discursive papers presented by people with an interest in a variety of topics that have been brought together under the general subtitle 'ethical issues in the new reproductive techniques'. The title gives the impression that the book contains a definitive account of the Baby $\boldsymbol{M}$ case incorporating carefully researched information about the experience of the sad protagonists in this cause célèbre, about the inadequacies of the judicial system asked to decide questions of parenthood and about the role of the state in a matter of intense individual expression in one of the most individualisticallycentred countries in the world. But what a disappointment! Only one short paper and an appendix are devoted directly to this topic, giving the impression that the case is being used rather than analysed.

The 14 essays are predictable and represent a wide cross-section of views of interested persons resident in the USA. This approach has disadvantages for a readership which has already been exposed to similar arguments and views at this general level in the UK; information which has experienced a long gestation period in its development. While the Baby $M$ case attracted worldwide attention, it should be remembered that Louise Brown, Baby Cotton and all the discussion and disputation surrounding the Warnock Report (1984) and the Human Fertilisation and Embryology Act (1990) have taken us far beyond an introduction to the subject matter. For me, at least, the book gives a powerful feeling of déjà vu, accompanied by regret that our American colleagues appear to read publications based on UK experience and argument less frequently than we do concerning theirs.

It's easy to carp; ten pages of historical reflection (under the title Science, Conscience and Public Policy no less!) which address abortion, contraception and the eugenics movement are followed by an essay which addresses the general concerns of six religious communities in the USA followed by reflections on the Jewish, Roman Catholic, Lutheran, Jehovah's Witness, Anglican, Greek Orthodox, Islamic and Eastern Orthodox perspectives of the new reproductive techniques - all in 18 pages. Interesting issues are raised but when they are, the desire to see them explored more fully is dashed in the interests of limited space. I agree with the sociologist Barbara Rothman when she says that 'we need to develop a technology for aiding procreation that reflects, and not destroys, our social relationships'. More discussion of this important issue would have been welcomed.

ROBERT SNOWDEN, Professor of Family Studies, Sociology Department, University of Exeter. 